Itinéraires Itinéraires

Littérature, textes, cultures

\title{
La banlieue côté femmes : une recherche-action à Gennevilliers (Hauts-de-Seine)
}

The Suburbs for Women: Action Research in Gennevilliers (Hauts-de-Seine)

\section{Corinne Luxembourg}

\section{OpenEdition}

Journals

Édition électronique

URL : http://journals.openedition.org/itineraires/3633

DOI : $10.4000 /$ itineraires.3633

ISSN : 2427-920X

Éditeur

Pléiade

Référence électronique

Corinne Luxembourg, « La banlieue côté femmes : une recherche-action à Gennevilliers (Hauts-deSeine) », Itinéraires [En ligne], 2016-3 | 2017, mis en ligne le 15 juillet 2017, consulté le 01 mai 2019. URL : http://journals.openedition.org/itineraires/3633 ; DOI : 10.4000/itineraires.3633

Ce document a été généré automatiquement le 1 mai 2019.

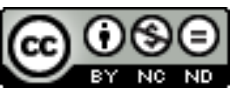

Itinéraires est mis à disposition selon les termes de la licence Creative Commons Attribution - Pas d'Utilisation Commerciale - Pas de Modification 4.0 International. 


\section{La banlieue côté femmes : une recherche-action à Gennevilliers (Hauts-de-Seine)}

The Suburbs for Women: Action Research in Gennevilliers (Hauts-de-Seine)

\section{Corinne Luxembourg}

\section{Introduction}

1 Depuis les années 1970, la politique de la ville, en France, a pour objet d'intervenir sur le bâti, l'urbanisme, les actions culturelles et sociales. Ces projets de rénovation urbaine tout comme l'urbain sur lequel ils interviennent sont autant le reflet de normes sociales dominantes, masculines que ne l'étaient les constructions précédentes. Les politiques publiques menées sont marquées par l'hétéronormativité de la structure familiale et l'androcentrisme des professions d'urbaniste, d'architecte et des acteurs publics. L'espace public serait ainsi alloué aux hommes et l'espace privé aux femmes tout comme la ville serait attribuée aux hommes, le logement aux femmes, la nuit aux hommes, le jour aux femmes. Cette assignation sexuée des espaces et des temporalités de l'habiter perdure et tend même, parfois, à se renforcer dans le même temps que la société dans son ensemble se replie sur elle-même. Malgré l'injonction au gendermainstreaming, défini par le Conseil de l'Europe comme «l'approche intégrée de l'égalité entre les femmes et les hommes [consistant] en la (ré)organisation, l'amélioration, l'évolution et l'évaluation des processus de prise de décision, aux fins d'incorporer la perspective de l'égalité entre les femmes et les hommes dans tous les domaines et à tous les niveaux, par les acteurs généralement impliqués dans la mise en place des politiques ", la construction de la ville, y compris de la ville durable, est la preuve d'une fixité certaine dans les normes masculines mobilisées.

2 Le 27 mars 2015, lors d'une journée d'étude organisée à Gennevilliers par Les Urbain.e.s ${ }^{1}$, dans le cadre de la recherche-action «La ville côté femmes », Claire Hancock rappelait les 
risques inhérents à la territorialisation de la question des inégalités femmes/hommes, notamment au renforcement de leur visibilité dans les espaces périphériques, particulièrement celui :

[...] d'exonérer le reste de la société, comme si les villes-centre et les beaux quartiers n'étaient pas aussi des hauts lieux de violence de genre et de discrimination à l'encontre des femmes et des minorités sexuelles. [...] Jacqueline Coutras soulignait que la visibilité des rapports de domination sexués, dans les quartiers dits sensibles, tenait au fait que, contrairement à ce qui se produit dans les quartiers plus favorisés, «les dominants n'ont pas les ressources suffisantes pour les organiser de façon à les rendre moins visibles $»^{2}$.

3 Mais le temps du processus de développement de la ville est un temps long, et l'espace public urbain est avant tout une hérédité, plus qu'un héritage que l'on pourrait rejeter en bloc. La construction de la ville des grands ensembles à la fin des années 1960 a répondu à plusieurs objectifs, en particulier l'impérieuse nécessité de trouver une solution à la situation de mal-logement tout en maintenant une relation forte entre le lieu de travail et le logement. Le zoning propre à la construction de la ville moderne de l'après Seconde Guerre mondiale séparant les fonctions urbaines a participé à compliquer la tâche des femmes et des mères désireuses de concilier rôles productifs et reproductifs en fractionnant les espaces et en les éloignant les uns des autres. Or, le travail de cette période reste encore majoritairement masculin, les carrières professionnelles féminines étant généralement écourtées, souvent suspendues à la naissance du premier enfant, ou se poursuivent à temps partiel.

4 Ces espaces urbains de « chemins de grue » dessinés et rapidement bâtis par les hommes sont l'espace vécu des femmes pour l'essentiel. Aujourd'hui, dans l'ensemble des villes occidentales, les femmes sont plus nombreuses que les hommes à vivre dans des villes construites par les hommes. Pour autant, si cela signifie que les femmes s'approprient un espace public urbain plus étendu, en se référant au nombre de lieux fréquentés, comme nous le verrons plus loin, cela ne se traduit pas par une occupation stationnaire des lieux comme peuvent le faire les hommes (bancs, halls d'immeuble, etc.), mais par une circulation où les arrêts sont rares et souvent conditionnés par des tâches familiales et/ ou domestiques, liées à un rôle reproductif. Enfin, cet habiter féminin est confronté aux déficiences générales de l'habiter de l'ensemble de la population dans nombre de ces quartiers de grands ensembles. Ainsi la fonction même d'habiter a été limitée à sa seule fonction d'abri, délaissant alors toute réflexion sur l'espace public comme espace vécu (Faure, Hernandez-Gonzalez, Luxembourg 2017).

5 Or, cette vie quotidienne du quartier comme espace vécu est souvent féminine. Les politiques urbaines ne se penchent sur la question féminine qu'au début des années 1980 en faisant des femmes non plus des occupantes silencieuses des quartiers mais des relais de la démarche de développement social des quartiers. Il s'agit, entre autres, d'encourager la formation et la professionnalisation. En effet, durant la décennie suivante les femmes accèdent plus nombreuses à l'emploi, bien que cet emploi soit souvent à temps partiel et à durée déterminée. Cependant, la lutte pour la place des femmes dans l'espace urbain est d'autant plus difficile qu'il s'agit d'une construction sociale du territoire « après coup » et qu'elle ne va pas encore de soi pour l'ensemble de la société, acteurs institutionnels compris, ceci dans un contexte où la conception de l'urbanisme est à repenser au prisme d'un habiter plus diversifié.

6 Un exemple est révélateur des déficiences de cet urbanisme moderne dont nous héritons : le travail non rémunéré. En effet, la ville fonctionnelle, en segmentant les espaces de 
logement, de récréation, de travail, de circulation, ne tient pas compte de temporalités où la coprésence des différentes typologies serait nécessaire. Ainsi les femmes se trouvent dans cette situation paradoxale : souhaitant s'émanciper du seul logement et du travail non rémunéré qu'elles assument majoritairement, leur statut de mère de famille les a longtemps contraintes à y demeurer ou au mieux à cumuler travail rémunéré et travail non rémunéré. La segmentation fonctionnelle ne fait alors qu'augmenter ce temps strictement alloué à la famille. Pourtant, la planification urbaine s'appuie encore souvent sur une logique spatiale et économique qui ne prend en compte que le travail rémunéré des personnes (et pas l'économie dite de reproduction ou du care) et se développe comme si chaque foyer comprenait nécessairement une personne restant à domicile.

7 En partant du paradigme selon lequel la division masculin-féminin sous-tend l'ensemble de l'organisation sociale, comme le fait l'urbaniste Lidewij Tummers (2015), la recherche «La ville côté femmes », conduite en partenariat avec la ville de Gennevilliers, s'intéresse aux pratiques quotidiennes de l'espace public en mêlant diverses méthodologies d'enquêtes, telles que des observations et des productions artistiques scéniques et photographiques.

\section{Lire les mobilités et les appropriations des espaces publics : cartes mentales et observations participantes}

$8 \quad$ Afin de prendre la mesure des usages des espaces publics par les femmes et les hommes, il s'est agi dans un premier temps d'envisager les mobilités de chacun. S'intéresser à la mobilité implique la prise en compte de ce qu'elle signifie. Synonyme de déplacement, elle s'élargit vers la facilité de se mouvoir (physique, sociale, etc.) incluant ainsi autant la réalité du déplacement que sa potentialité. Ainsi, la mobilité géographique pourrait être envisagée comme un indicateur de capital économique, social, culturel autant qu'un indice d'émancipation. De là découle une forme d'injonction à la mobilité liant la mobilité sociale à la mobilité physique, où l'intensité est associée à une promotion sociale.

Néanmoins, si l'une et l'autre sont indiscutablement liées, la traduction politique qui en est faite, peut être discutée, d'autant plus lorsqu'il s'agit d'observer les mobilités féminines dans un contexte où la mobilité sociale a de plus en plus à voir avec une situation de précarité sociale. En effet, la mobilité est devenue une caractéristique du travail rémunéré.

10 La mobilité urbaine concerne donc cette interface entre moyen d'émancipation et obligation sociale, mais aussi entre transit et arrêt. Pour ce qui concerne notre travail présenté ici, d'une part les femmes seraient donc encore prises entre l'injonction domestique (et donc à la mobilité) à poursuivre leur travail de mère en dehors du domicile familial pour s'occuper des activités scolaires et extrascolaires des enfants, et pour nombre d'entre elles pour ravitailler le foyer, et l'immobilité contrainte dans le cadre de l'espace privé. D'autre part, conséquence de cette première assertion, les femmes sont plus mobiles que les hommes. C'est ce qu'indique la collecte de cartes mentales produites par les habitants et les habitantes de Gennevilliers. 


\section{Collecte de cartes mentales}

11 Partant de l'hypothèse que le corps est le premier vecteur de son positionnement dans l'espace, la représentation de sa spatialisation peut aussi être vectrice d'utilisation plus large de l'espace public. Il a l'avantage de rendre plus lisibles les « marqueurs spatiaux ».

Les cartes mentales associent la connaissance que les gens ont de leur environnement et leur expérience par la pratique de cet environnement. L'hypothèse que nous formulons est que plus les cartes sont fournies, présentant des repères spatiaux, plus leur auteur connaît le territoire communal et le pratique. Ces cartes permettent aussi d'aller au-delà d'un simple relevé physique des déplacements, et donnent des indications quant à la perception des espaces. Les distorsions, la liberté vis-à-vis des échelles de représentation classiques, informent sur des contenus sensoriels (vue, audition, odorat, etc.) et émotionnels. Les mémoires, les choix, conscients ou non sont une simplification et une accumulation de ces différents éléments. En outre, par leurs décisions, les cartographes agissent sur leur espace, comme celui-ci agit sur eux. C'est-à-dire que l'expression de leurs déplacements, de leurs pratiques de l'espace public peut induire, ou renforcer, une perception de l'espace, un regard collectif.

Fig. 1. Exemple de carte mentale

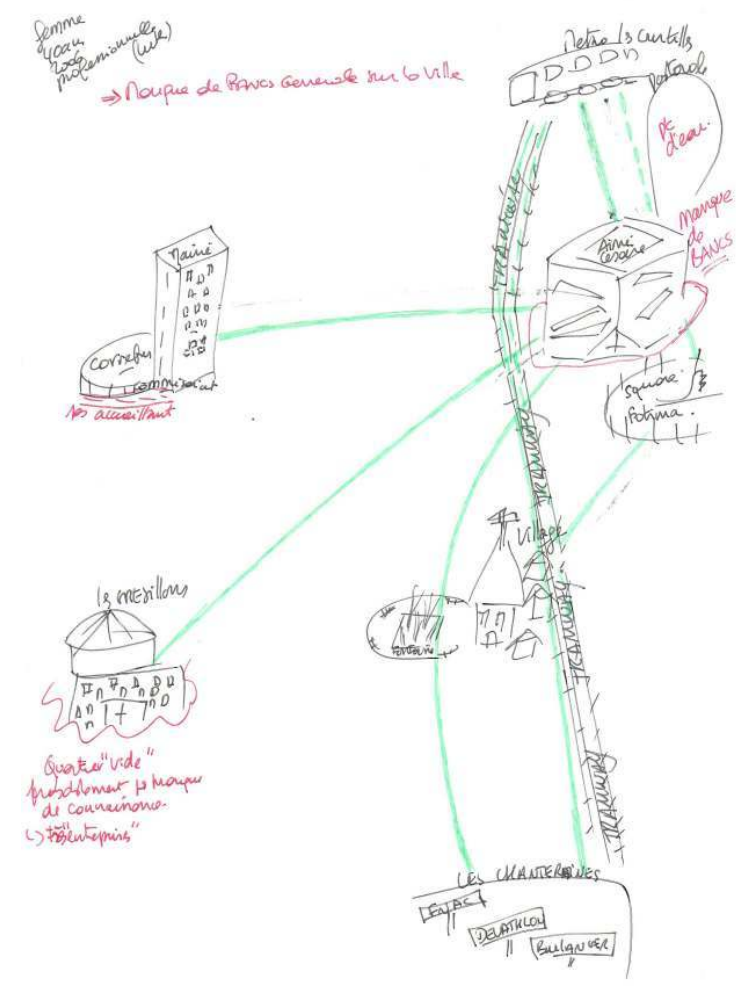

Enfin, il est indispensable de noter que ces cartes ne sont pas stabilisées. Elles sont sans aucun doute différentes selon l'âge, l'expérience, le sexe, les activités, le ou les groupes sociaux, mais elles évolueront aussi dans le temps, selon l'environnement lors du dessin. Pour les analyser, nous avons donc réalisé des grilles pour compiler les informations et des cartes de synthèse transposant les données de chacune des cartes mentales collectées. 
14 Avec un minimum d'éléments de contextualisation (âge, date d'arrivée à Gennevilliers, quartier de résidence ou de travail) et une feuille blanche, nous avons demandé aux hommes et aux femmes de représenter leurs déplacements dans la commune en différenciant les parcours appréciés en vert, les parcours sans intérêt (ni aimés ni dépréciés) en noir, les parcours faisant l'objet d'un malaise, d'une appréhension, d'une méfiance en rouge. En trait plein sont dessinés les trajets de jour, en pointillé les trajets de nuit, en variant les couleurs selon le ressenti. Ce protocole a été mis en œuvre à la mijuin 2014 auprès du petit échantillon de femmes de l'École Femmes sans frontières, puis en septembre 2014, lors de la journée du forum des associations où il a été réalisé par plus de 60 personnes, enfin en mars 2015 à l'occasion de la journée internationale des droits des femmes, où une trentaine de personnes ont dessiné leurs trajets.

15 L'analyse de 102 cartes également réparties entre hommes et femmes tend à montrer que les femmes s'approprieraient plus largement le territoire communal que les hommes. $\mathrm{Si}$ ceux-ci le parcourent sans doute, ils ne représentent et ne citent que peu de quartiers sur les huit de la commune.

16 Pour l'analyse de chacune des cartes, est compté le nombre de quartiers représentés. On observe alors trois éléments :

- Plus de femmes que d'hommes ne représentent ou bien aucun quartier ou bien que leur seul quartier de résidence. Les hommes sont bien plus nombreux à dessiner deux quartiers. Ce premier constat représente un tiers de l'échantillon aussi bien masculin que féminin. Ainsi, un tiers des femmes qui se sont pliées à l'exercice de la carte mentale, ne s'approprient que peu la ville et ne dépassent que rarement le périmètre du quartier de résidence.

- Un deuxième tiers montre que pour fréquenter 3 quartiers, les femmes sont aussi mobiles que les hommes et s'approprient autant ce périmètre qui correspond selon Michel de Certeau (1990) à une moyenne des parcours piétons réalisés.

- Pour citer 4, 5, 6 et 8 quartiers (sur les 8 quartiers que compte la ville), les femmes sont beaucoup plus nombreuses. Ce qui tendrait à indiquer que pour ce dernier tiers des femmes qui ont réalisé la carte mentale, elles s'approprieraient plus la ville dans son ensemble en y effectuant beaucoup plus de trajet. Elles dépassent beaucoup plus souvent que les hommes les « limites » des espaces du quartier de résidence. 
Fig. 2. Appropriation du territoire gennevillois par les hommes et les femmes (relevé à partir des cartes mentales récoltées auprès de personnes habitant la ville).

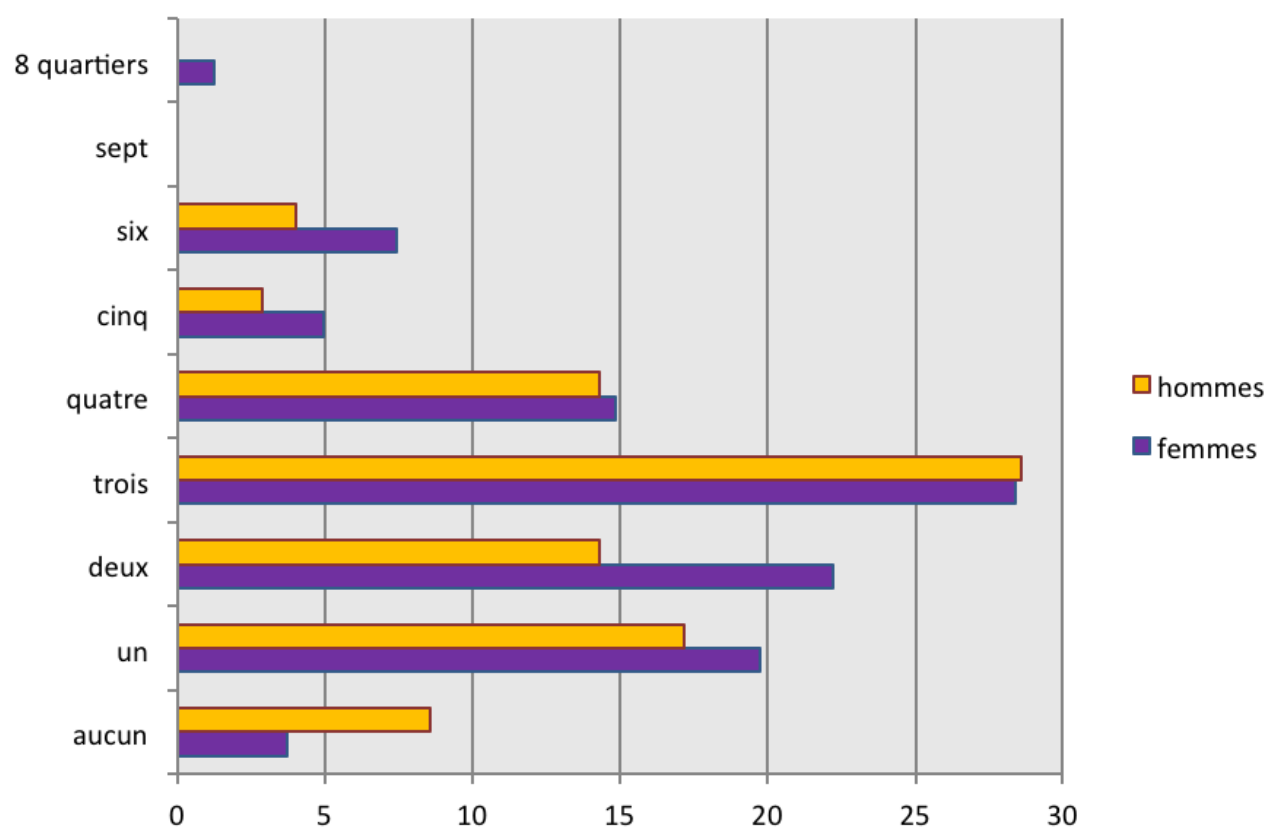

La répartition des lieux fréquentés donne sur ce point des indications intéressantes : les femmes en nomment beaucoup plus. Ce qui est cohérent avec le fait qu'elles sont plus nombreuses que les hommes à fréquenter des espaces plus larges. La précision des lieux cités est plus grande sur les cartes dessinées par les femmes que sur celles dessinées par les hommes.

Elle correspond aussi à des pratiques de la ville qui sont plus liées à des tâches domestiques et familiales pour les femmes que pour les hommes. Ainsi, même s'il est indubitable que des hommes fréquentent les commerces, notamment les centres commerciaux, ils ne les citent quasiment jamais. Les usages des espaces végétalisés sont fréquemment liés à l'accompagnement des enfants pour les femmes tout comme le sont les usages des équipements sportifs. On retrouve une fréquentation des transports publics plus importante par les femmes que par les hommes, observation notée dans d'autres travaux scientifiques et sur d'autres villes ${ }^{3}$.

Enfin, la synthèse cartographiée des données spatialisées recueillies sur les cartes mentales donne des éléments sur les pratiques et sur les représentations. Par exemple, aucune des femmes ayant dessiné la rue Georges Thoretton ne l'apprécie : quand elle est jugée par des femmes, c'est uniquement négativement. Ce jugement ne concerne que la fraction de la rue du côté de l'avenue Gabriel Péri. Il se passe exactement la même chose pour la rue Jean Jaurès, ainsi que pour la rue Pierre Timbaud. Les raisons évoquées sont autant liées à des commerces vus comme très masculins (cafés, par exemple), qu'à une ambiance esthétique (tristesse d'un lieu méritant réhabilitation, murs trop longs), des difficultés de circulation (à pied, avec des enfants, à vélo, etc.) ou un sentiment d'insécurité routière. À l'inverse, c'est le boulevard intercommunal en direction du port qui n'est mentionné et jugé négativement que par les hommes, du fait d'un aspect trop adapté à la circulation automobile rapide et pas assez aux piétons. 
20 Pour l'ensemble de ces résultats, il est nécessaire de ne pas associer de façon systématique le fait de ne pas aimer un lieu et le fait de s'y sentir en insécurité. Ainsi sur les cartes dessinées par les femmes comme par les hommes, des commentaires donnent plus d'indications sur des aspects esthétiques (la dalle de Carrefour, l'avenue de la Libération, la rue Jean Jaurès, le métro Gabriel Péri, etc.), d'ambiance climatique (le métro des Courtilles est froid, celui des Agnettes en courant d'air), ou liés à l'accessibilité et à la praticité (trottoirs trop petits, trottoirs trop pentus, l'accès possible aux vélos), et aux pratiques et usages détournés (voitures garées sur le parcours du tramway, garées sur le trottoir empêchant le passage de poussettes ou de fauteuils roulants, propreté des trottoirs). Arrivent enfin les questions de sécurité routière (avenue Gabriel Péri, vers le pont de Clichy ou le pont de Saint-Ouen) et le sentiment d'insécurité lié à un faible éclairage de nuit et à une faible présence humaine (les Barbanniers). La relation du corps de la femme ou de l'homme à l'espace urbain est aussi explicitement mentionnée, en rapport aux voitures par exemple, aux cheminements sur les trottoirs, à la longueur de certains bâtiments trop uniformes, etc.

Hommes et femmes notent facilement des remarques sur l'environnement, l'entretien de la voirie. Les hommes ajoutent souvent des commentaires en rapport avec leurs sensations. Les femmes n'en font jamais état par écrit, alors qu'elles peuvent les exprimer lors d'entretiens. Cette différence sexuée du vécu des espaces publics urbains corrobore ce que les travaux des géographes Doreen Massey en 1984, puis Jacqueline Coutras en 1996, ont permis d'affirmer : la ville peut être lue comme un espace construit par des rapports sociaux de sexe qui fluctuent en fonction des contextes culturels et sociaux et il existe des itinéraires sexués différenciés. Ces territorialités révélées par les mobilités sont une expression de la division sexuée du travail, dans les rythmes, les parcours et les activités (Tummers 2015, Coutras 1996).

La prise en compte de la différenciation sexuée des usages de la rue - circulatoire pour les femmes, statique pour les hommes - entre peu à peu dans les décisions politiques. Néanmoins, tout comme les recherches sur la ville, les travaux restent majoritairement locaux. Ainsi, de la même façon que la femme serait plutôt assignée à l'espace privé, à la vulnérabilité, elle serait aussi cantonnée à l'échelle locale.

Ce premier temps permet de récolter des informations sur l'appropriation des espaces publics, des territoires dans leur ensemble, mais rend peu compte des temporalités de chacun et chacune, et des co-présences éventuelles. Cela est principalement l'objet des observations photographiques.

\section{Observations photographiques}

Pour analyser la présence des femmes et des hommes dans l'espace, nous avons construit une sorte de cartographie-photographie inspirée de l'œuvre de Gérard Fromanger: Hommage à François Topino-Lebrun. Cette série en hommage au peintre révolutionnaire guillotiné en 1801 reprend l'œuvre qui le fit remarquer, La Mort de Caïus Gracchus et la décline en cinq variations : La Mort de Caïus Gracchus (1975-1977), La Vie et la Mort du peuple (1975-1977), La Mort de Pierre Overney (1975), Le siège de Lacédémone par Pyrrhus repoussé par les habitants eux-mêmes de tout sexe et de tout âge (1989), La Vie des idées (1975-1977). Les deux premiers tableaux constituent un diptyque sur lequel nous nous appuyons ${ }^{4}$. Il donne une légende de cartographie commune à la lecture de deux images et de deux événements. Le premier évoque l'assassinat du tribun de la plèbe Caïus Gracchus en 
121 av. J.-C. après avoir voulu attribuer la citoyenneté aux Latins et Italiens de la colonie de Carthage et tenté une réforme agraire permettant aux plus pauvres d'améliorer leur condition sociale. Le second fait référence au meurtre du militant de la Gauche prolétarienne Pierre Overney en février 1972 après avoir incité les ouvriers de l'usine de Renault Billancourt à commémorer le massacre de Charonne de février 1962.

L'intérêt est ici multiple. Il s'agit de représenter les personnes de tout sexe et de tout âge présentes dans l'espace urbain, mais aussi les équilibres et les déséquilibres apparents, de viser la mixité comme moyen de plus grande citoyenneté et d'émancipation. La méthodologie consiste à cartographier sur la photographie les hommes et les femmes ; carte, puisqu'il s'agit de représenter des données localisées.

Le transfert méthodologique permet de coloriser les personnes photographiées sur des sites sélectionnés. Les deux exemples choisis de cette carto/photographie correspondent à un dimanche après-midi autour d'un petit centre commercial et d'un café (photo de gauche), et à un mercredi matin à la sortie de l'école (photo de droite).

Fig. 3. Occupation de l'espace public, un dimanche à 14 heures devant un café et un mercredi à 11 heures devant une école. Janvier 2015.
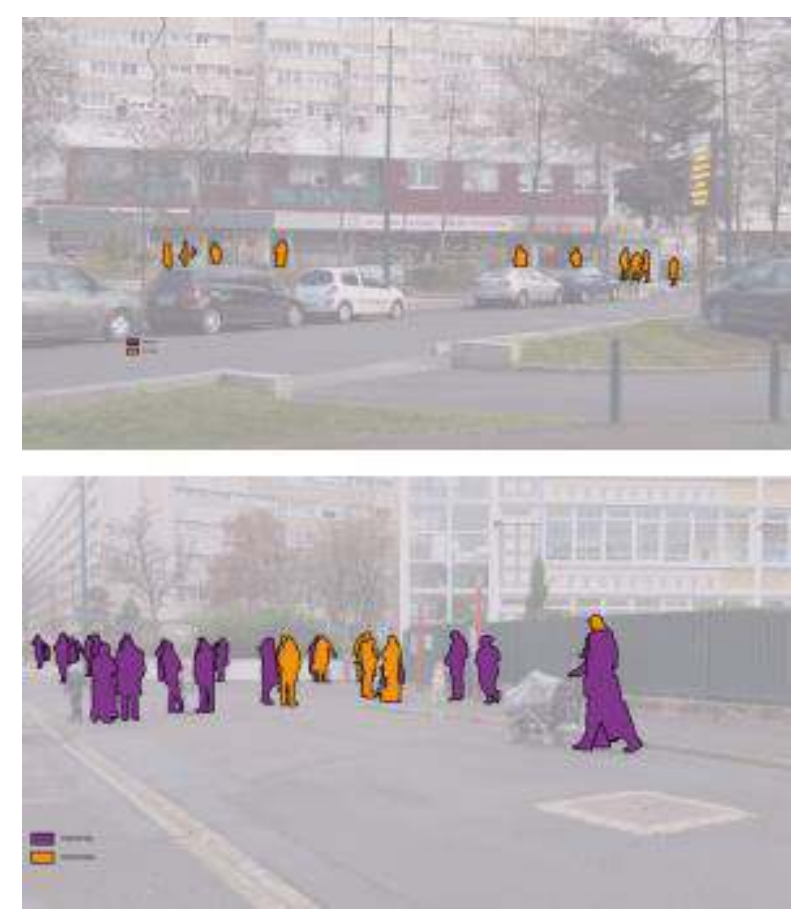

Sont représentés en jaune les hommes, en violet les femmes.

Les hommes sont minoritaires devant l'école et ont une présence exclusive sur le trottoir devant le café. Plusieurs lieux sont ainsi repérés comme étant des lieux de circulation ou d'arrêt. Ce qui caractérise l'arrêt des femmes est un prétexte familial: l'enfant à accompagner à l'école par exemple, à surveiller au parc ou dans les aires de jeux, etc., bien plus que l'arrêt des hommes qui est lié à des temps de repos, de rencontre, de sociabilité. Nous pouvons interroger le caractère effectivement public du segment de trottoir utilisé, non pas dans son cadre juridique, mais bien dans son usage. La suite du programme permettra de généraliser la méthode pour prendre en compte une diversité de lieux de manière synchronique et diachronique. 

psychologie spatiale développée par Abraham Moles ([1998] 2009) dans une logique de phénoménologie. Cette approche transversale permet d'aborder l'individu dans sa globalité, c'est-à-dire sa façon de penser l'espace et les stratégies qu'il met en œuvre pour tirer profit des structures de l'espace. L'approche initiale est celle de la polarité entre l'ici et l'ailleurs, entre l'habiter et l'étranger, entre le connu et l'inconnu. L'idée de l'appropriation de l'espace par l'individu est donc centrale avec comme corollaire un gradient progressif du plus approprié au moins approprié. La structure élémentaire du territoire individuel correspond à un emboîtement d'espaces, telles des poupées russes. Ce sont les coquilles de l'espace et l'individu est au centre de son espace. L'hypothèse de la proxémique est que l'individu perçoit plus précisément ce qui est proche et que l'importance des événements et des choses décroît avec la distance. Il sera de fait particulièrement sensible aux éléments spatiaux qui vont structurer son quotidien. Pour notre sujet, la perception de l'Autre est primordiale car de cette perception découle des comportements de domination ou d'évitement spatial, de mise en relégation ou au contraire de survalorisation de certains espaces par des pratiques quotidiennes de l'habiter.

Ainsi, certains lieux pourraient être considérés comme une excroissance du domaine privé sur le domaine public. Ils sont ceux appropriés par les femmes dans leur rôle de mère et où les pratiques sociales, qui s'y déroulent, permettent un entre-soi accentué (Moullé 2013). À l'opposé, les abords des cafés rassemblant un groupe d'hommes se diluent dans l'espace public en excluant les femmes par des remarques, des attitudes exclusives et remettant en cause les possibilités d'appropriation pour la moitié de la population. Les lieux de circulation permettent une plus grande mixité, mais l'appropriation est nettement plus faible, sauf pour ceux qui y stationnent par nécessité comme les personnes sans logis.

À cette première base résultant d'études urbaines, s'ajoutent des approches artistiques, notamment par le biais d'un atelier d'écriture féminin (de septembre 2015 à juin 2016), d'ateliers théâtraux intergénérationnels mixtes (2016-2018) et d'interventions chorégraphiées.

31 Ces situations diverses permettent d'interroger collectivement les analyses souvent binaires pour parvenir à l'émergence d'une hybridation des méthodologies de recherche, mais aussi d'un tiers espace. Ce tiers espace, c'est une autre manière de faire collectif entre identité et altérité.

\section{Échanges scientifiques et artistiques}

L'ensemble de ce travail sert de point de départ à l'échange avec différents artistes intervenant sur la commune de Gennevilliers, pour la plupart associés au déroulement du projet, pour quelques autres non.

33 C'est le cas d'une œuvre déjà existante comme Mademoiselle, pièce chorégraphiée de la compagnie Jeanne Simone et représentée en avril 2015 dans les rues de Gennevilliers avec le soutien du service culturel de la ville.

Deux représentations ont eu lieu, l'une le midi, l'autre en soirée. La Maison du développement culturel (MDC) a envoyé à une liste de personnes familières de ce type de performances, un mot de passe indiquant le lieu de rendez-vous. Sur place, le public

Itinéraires, 2016-3 | 2017 
découvre Monsieur, qui connaît Mademoiselle et a pris l'habitude de l'observer sur son chemin quotidien. Monsieur invite les spectateurs à une sorte de filature collective et les accompagne vers le premier point d'observation, en face du lieu de travail de Mademoiselle. Le public doit la repérer sur ce lieu de travail, au milieu de ses collègues, dans son intégration dans le réel, le quotidien. Ses gestes sont d'abord ceux, appropriés, de l'employée et sa posture est fonctionnelle. Petit à petit, elle décale physiquement ses actions (répétitions, arrêts sur image, etc.), ce qui la distingue alors aux yeux des spectateurs. Elle quitte son travail, talonnée par Monsieur qui sert de guide dans l'espace public ou semi-public 5 .

Différentes facettes de cette femme se révèlent. D'un vêtement à l'autre, elle se transforme durant le parcours qui fait sa journée. Les différents points de vue proposés par Monsieur aux spectateurs, les changements de rythme dans ses déplacements, le jeu de dédale auquel il les convie au sein d'un quartier, permettent d'apprécier de façon sensitive la manière d'être au monde de Mademoiselle ${ }^{6}$.

À la fin du parcours, Mademoiselle disparaît lentement, offrant une longue perspective dans le champ de vision du spectateur. Monsieur, quant à lui, s'en retourne à sa vie.

Fig. 4. Représentation de Mademoiselle dans le quartier du village à Gennevilliers en avril 2015.

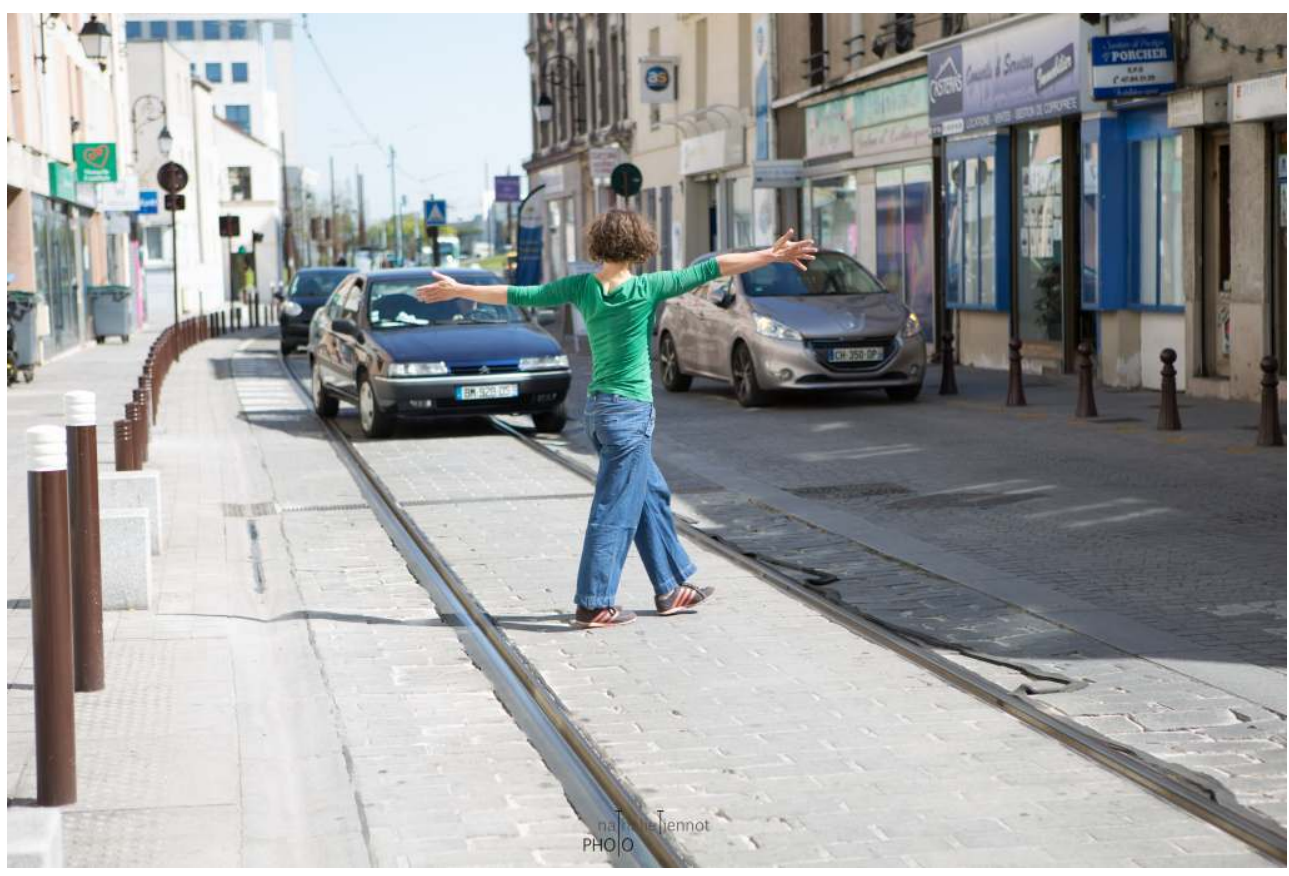

Photographie de Nathalie Tiennot, https://nathalietiennot.com/

L'écriture de Mademoiselle préexiste à la recherche-action « La ville côté femmes » menée par les Urbain.e.s à Gennevilliers, mais la participation à la représentation de cette œuvre vient compléter la réflexion cherchant à mettre en relation les formes urbaines et mêlant les déplacements des corps humains dans leur quotidienneté. Catherine Grout, dans L'Horizon du sujet (2012), met en évidence le rapport politique des présences des corps à l'espace :

La «pertinence politique de la question du sujet » (Michel de Certeau) trouve ici un de ses fondements, dans l'absolue nécessité de considérer le sujet comme faisant partie du monde et non comme une donnée objective, invariable et extramondaine. En prenant en compte mon expérience corporelle et ma manière d'être là dans l'espace commun, c'est-à-dire de m'y mouvoir mais aussi de m'y présenter, 
de m'y placer et de m'orienter par rapport et avec autrui, j'aborde à la fois la condition humaine, ma co-présence avec autrui, la pluralité, la différence et l'apparaître commun ainsi que l'horizon que je porte en moi et auquel je me relie dans un renouvellement permanent. Si l'analyse du politique par le sentir s'impose alors comme une évidence, elle réclame l'expérience et la reconsidération de l'espace par la spatialité. (Grout $2012: 14$ )

C'est ce même rapport que les participantes de l'atelier d'écriture « Raconter la ville au féminin", conduit par Damien Labruyere de septembre 2015 à juin 2016, ont fait apparaître dans leurs textes lus en public le 27 juin 2016.

Fig. 5. Lecture des textes de l'atelier « Raconter la ville au féminin », le 27 juin 2016.

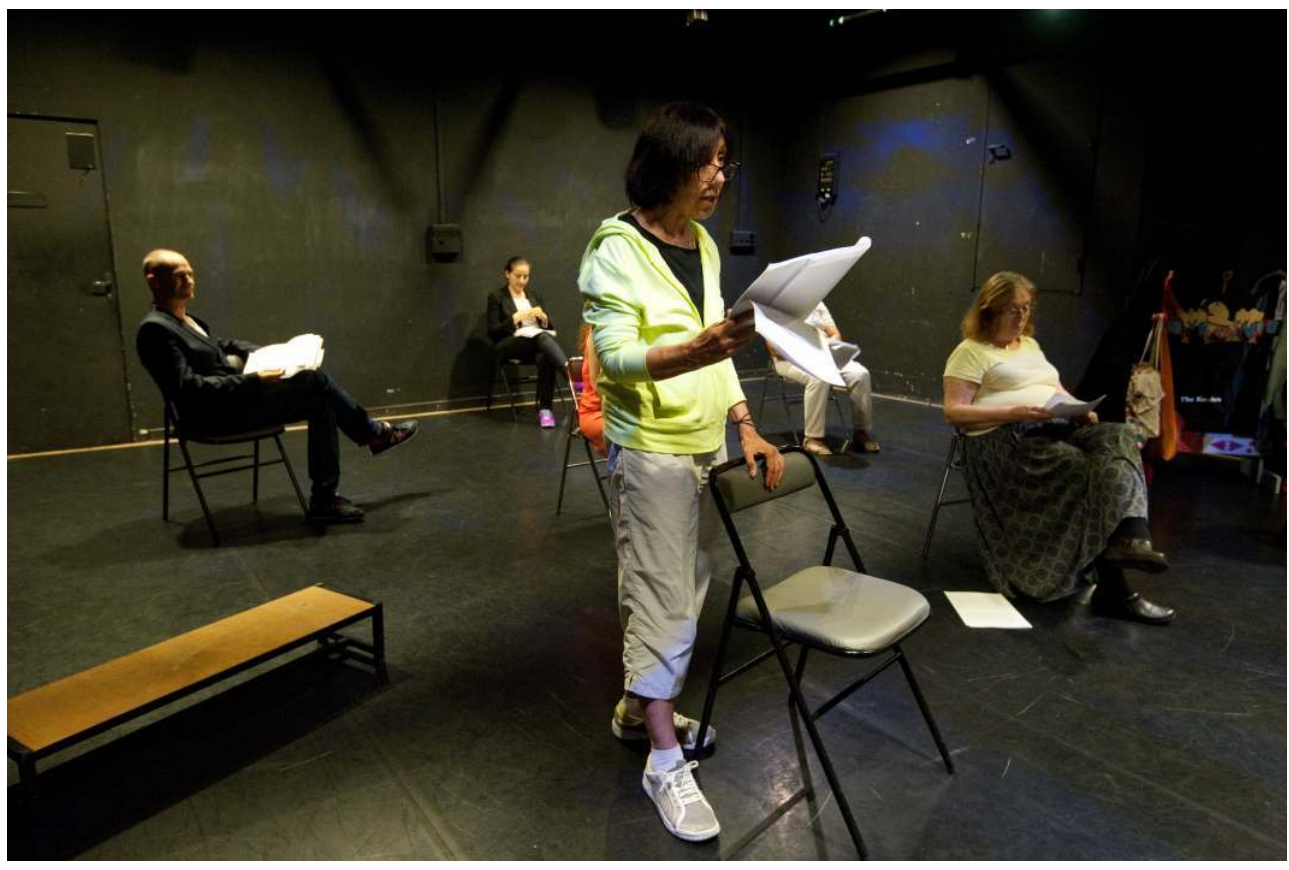

(c) Guillaume Clément.

37 L'atelier est orienté principalement sur le rapport des femmes à l'espace urbain, sur les plus ou moins grandes difficultés de coprésence femmes-hommes dans l'espace public (à défaut peut-être de mixité). Les textes expriment les difficultés d'occuper de façon statique certains lieux, comme des bancs, s'il n'y a pas de justification fonctionnelle à cette présence (surveillance d'enfant par exemple) : «S'asseoir sur un banc toute seule, même si c'est une invite à se prélasser impose d'avoir une occupation. Se libérer du regard de l'autre semble un exercice de haut vol!", écrit Harriette, l'une des participantes. Tout comme le regard discriminant sur les territoires de banlieue qui vient s'ajouter aux habituelles thématiques de l'intersectionnalité (sexe, race, classe, etc.). C'est ce que rappelle Nathalie, autre membre de l'atelier :

En arrivant sur Gennevilliers il y a dix ans, j'imaginais débarquer dans une ville de banlieue avec des barres, enfin les trucs glauques des reportages à sensation... Du coup en découvrant ces petites maisons avec des petits passages piétons j'ai toujours l'impression d'être dans un petit hameau au milieu de la ville.

Les textes ${ }^{7}$ issus de cet atelier balaient les pratiques de l'espace vécu. Christiane énonce «la rue, c'est pas pour les filles!», Agnès raconte les rencontres d'enfants, de sorties d'école, Latifa des souvenirs de retour de nuit, etc. 

mettant en espace différents profils de femmes et d'hommes sur une place, interagissant peu ou prou les un.e.s avec les autres. Si le premier atelier donnait à entendre le regard des femmes sur la ville et ce que le paysage urbain suscitait en elles comme émotions, le deuxième donnait plutôt à voir des frottements, des rencontres ou des solitudes, des regards d'individus sur d'autres pour enfin décider de construire un collectif favorisant le droit à la ville.

Fig. 6. Représentation de la pièce Une place à soi, le 22 juin 2017. Direction et mise en scène Damien Labruyere.

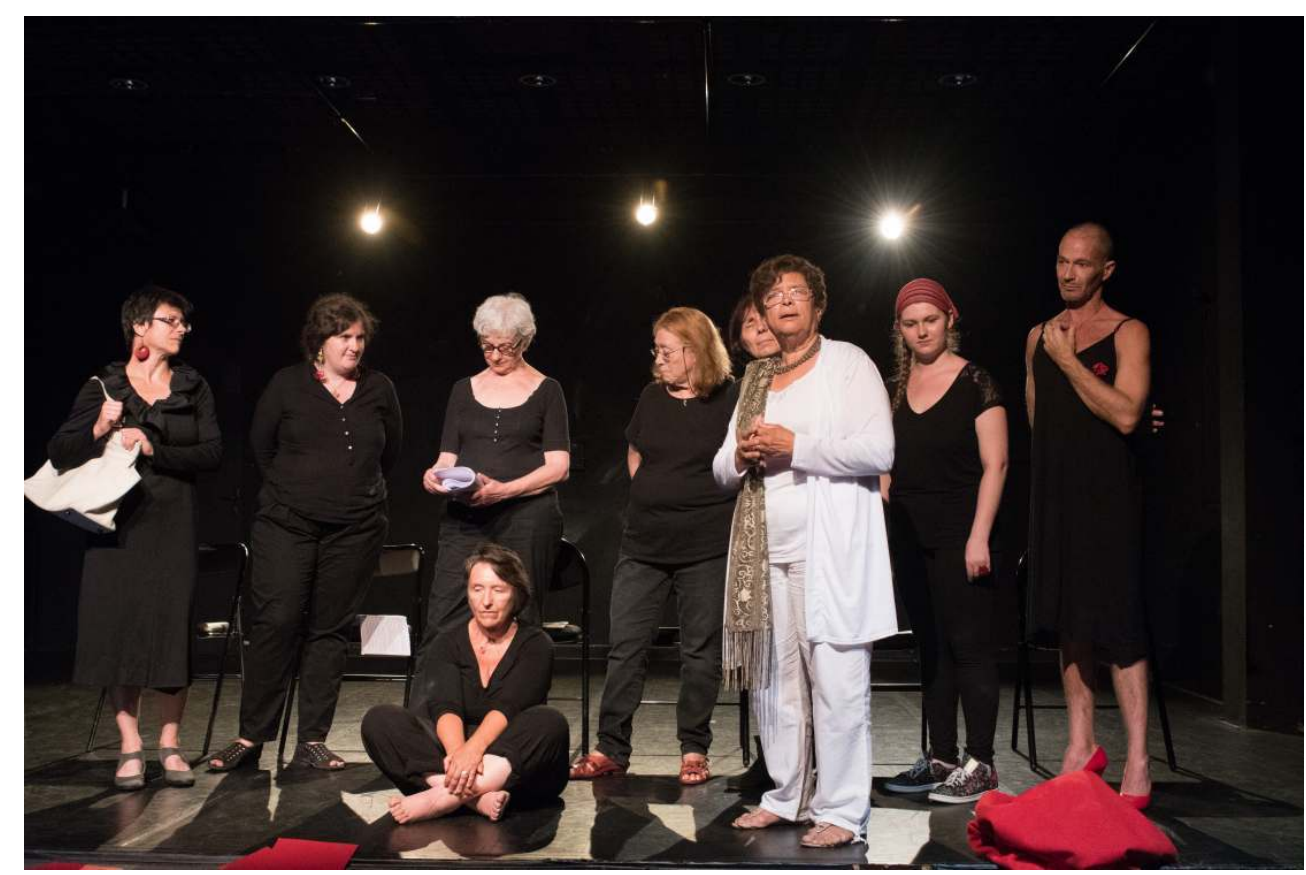

(c) Guillaume Clément.

La médiation permise par ces actions culturelles participe à construire un contexte favorable à une recherche-action participative. En effet, en associant les habitantes et les habitants, les ateliers sont un lieu de prise de conscience et de modification des comportements, des conceptions des rapports genrés dans l'espace, et les résultats de la recherche-action fournissent un diagnostic partagé aux différents services de la collectivité territoriale. Ces deux pans des travaux font d'un territoire de banlieue comme Gennevilliers un espace de visibilités et d'actions sur le genre. Il est enfin intéressant de noter que ce travail collectif qui, au départ, ne devait qu'alimenter la recherche-action, a donné naissance à une sorte de troupe amateur dont l'axe de réflexion principal est le rapport du corps (notamment féminin) à l'espace.

\section{Conclusion}

41 Saisir les rapports de genre à l'espace par la vie quotidienne est sans doute le plus complexe. Le travail avec les artistes, surtout en ce qui concerne les aspects intimes, les sens, le corps, a à voir avec l'esthétique des espaces publics et le ressenti des habitantes et habitants. 
Il s'agit d'aller plus avant dans l'étude des politiques locales en interrogeant la manière dont les actions féministes peuvent être un champ d'expérimentation dans les politiques d'aménagement et d'urbanisme. Par le biais de cette réflexion, nous avons souhaité aborder les politiques urbaines par le prisme féminin en questionnant nombre de comportements, de qualifications, de stéréotypes, de rapports sociaux de sexe, longtemps restés impensés parce qu'ils reposent, pour l'essentiel, sur des présupposés de détermination naturaliste. Il s'agit alors, par le biais du projet de recherche ici présenté, de rendre pérenne un questionnement permanent sur la ville, sur ses espaces, ses rythmes, ses usages. La démarche appelle à la remise en cause des certitudes et doit aussi laisser la trace d'un apprentissage commun et réciproque enrichissant. La politique de mixité dépend des envies et des besoins des habitant.e.s de vivre leur ville. Or, ces besoins dépendent eux-mêmes d'éléments combinés (emploi, âge, CSP, histoire, etc.). Ainsi, si l'on considère que l'augmentation des mobilités urbaines permet une meilleure appropriation de la ville, une diversification des modes d'habiter, on peut imaginer une réciproque qui serait que la conscience d'appropriation d'un territoire plus importante entraîne mécaniquement le bien-être dans un territoire toujours plus étendu et la mobilité hors des murs, favorisant ainsi la fonction émancipatrice de la ville.

\section{BIBLIOGRAPHIE}

Certeau, Michel de, 1990, L'Invention du quotidien : arts de faire, Paris, Gallimard.

Coutras, Jacqueline, 1996, Crise urbaine et espaces sexués, Paris, Armand Colin.

Denèfle, Sylvette (dir.), 2008, Utopies féministes et expérimentations urbaines, Rennes, PUR.

Faure, Emmanuelle, Hernandez-Gonzalez, Edna et Luxembourg, Corinne, 2017, La Ville : quel genre? L'espace public à l'épreuve du genre, Montreuil, Le Temps des Cerises.

Grout, Catherine, 2012, L'Horizon du sujet : de l'expérience au partage de l'espace, Paris, La lettre volée.

Hancock, Claire, 2015, « “Territorialiser” les politiques d'égalité femmes-hommes, pour quoi faire? », [En ligne], https://f.hypotheses.org/wp-content/blogs.dir/1746/files/2015/04/«Territorialiser-»-les-politiques-d'égalité-femmes.pdf.

Massey, Doreen, 1984, Spatial Divisions of Labour: Social Structures and the Geography of Production, New York, Methuen.

Moles, Abraham et Rohmer, Élisabeth, [1998] 2009, Psychosociologie de l'espace, éd. complétée, Paris, L'Harmattan.

Moullé, François, 2013, « La frontière et son double », Belgeo, nº 1, [En ligne], http://

belgeo.revues.org/10620

DOI : 10.4000/belgeo.10620.

Segaud, Marion, 2007, Anthropologie de l'espace. Habiter, fonder, distribuer, transformer, Paris, Armand Colin. 
Tummers, Lidewij, 2015, « Stéréotypes de genre dans la pratique de l'urbanisme », Travail, genre et sociétés, $\mathrm{n}^{\circ} 33$, p. 67-83.

\section{NOTES}

1. https://urbaines.hypotheses.org/. Les Urbain.e.s est un collectif de chercheuses et de chercheurs en études urbaines (géographes, sociologues, urbanistes, architectes, politistes), d'artistes (spectacle vivant et photographe), d'habitantes et d'habitants. Ses travaux font l'objet de restitutions et de médiatisations par le biais de créations culturelles (expositions, lectures publiques, créations théâtrales, sonores, etc.) et scientifiques (colloques, publications d'ouvrages, etc.).

2. Claire Hancock, "Territorialiser " les politiques d'égalité femmes-hommes, pour quoi faire?, [En ligne], https://f.hypotheses.org/wp-content/blogs.dir/1746/files/2015/04/«-Territorialiser-»les-politiques-d'égalité-femmes.pdf.

3. Par Yves Raibaud ou Arnaud Alessandrin et Johanna Dagorn à Bordeaux par exemple, ou par Sylvette Denèfle dans l'introduction de Utopies féministes et expérimentations urbaines (Denèfle 2008).

4. Les œuvres sont visibles au Centre Pompidou à Paris depuis 2013: https:// www.centrepompidou.fr/cpv/resource/cbqLK7a/rpxobX.

5. Dossier de présentation de Mademoiselle, [En ligne], http://www.jeannesimone.com/wpcontent/uploads/2015/06/Mademoiselle_DOSS-DIFF_Jeanne-Simone_SignatureG.pdf.

6. Ibid.

7. La première lecture publique de l'atelier est disponible : https://urbaines.hypotheses.org/414.

\section{RÉSUMÉS}

Aborder la banlieue par le genre renvoie assez systématiquement à un espace public autorisé qui se restreint sous la force tacite ou explicite du contrôle social patriarcal mais également à des situations d'insécurité, de violences, etc. Les espaces urbains dessinés et bâtis par les hommes sont l'espace vécu des femmes pour l'essentiel. Aujourd'hui, dans l'ensemble des villes occidentales, les femmes sont plus nombreuses que les hommes à vivre dans des villes construites par les hommes. Pour autant, si cela signifie que les femmes ont une plus grande appropriation de l'espace public urbain, en se référant au nombre de lieux fréquentés, cela ne se traduit pas par une occupation pérenne des lieux comme peuvent le faire les hommes (bancs, halls d'immeuble, terrasses de café, etc.), mais par une circulation où les arrêts sont rares et toujours conditionnés. Dès lors, comment envisager «l'espace vécu » féminin ? Le travail de recherche-action développé à Gennevilliers s'organise autant autour de méthodologies classiques de terrain que d'ateliers artistiques participatifs (ateliers d'écriture notamment).

Studying the suburbs by gender refers systematically to an authorized public space restricted by the tacit or explicit force of the patriarchal social control but also to situations of insecurity, violence, etc. Urban spaces designed and constructed by men are essentially the living space of women. Today, in all Western cities, more women than men live in cities built by men. However, if this means that women have a greater appropriation of the urban public space, by referring to 
the number of places frequented, this does not translate into a perennial occupation of the places as men can do (schools, Halls of building, terraces of coffee, etc.), but by a circulation where the stops are rare and always conditioned. Hence, how can we envisage the "living space" of women? The research-action program developed in Gennevilliers is organized around classical field methodologies as well as participatory artistic workshops (writing workshops, etc.).

INDEX

Mots-clés : genre, espace public, espace vécu, ateliers artistiques participatifs, Gennevilliers Keywords : gender, public space, lived space, participatory artistic workshops, Gennevilliers

\section{AUTEUR}

\section{CORINNE LUXEMBOURG}

Université d'Artois, Centre de recherche Discontinuités (EA 2468) 